\title{
Programmable cell-free transcriptional switches for antibodies detection
}

Aitor Patino, ${ }^{[a], \#}$ Sara Bracaglia, ${ }^{[a], \# ~ S i m o n a ~ R a n a l l o, ~}{ }^{[a]}$ Tania Patino, ${ }^{[a]}$ Alessandro Porchetta, ${ }^{[a]}$ and Francesco Ricci [a],*

[a] Department of Chemistry, University of Rome, Tor Vergata, Via della Ricerca Scientifica, 00133 Rome, Italy,

*Corresponding author: francesco.ricci@uniroma2.it

\# These authors contributed equally

\begin{abstract}
We report here the development of a cell-free in-vitro transcription system for the detection of specific target antibodies. The approach is based on the use of programmable antigen-conjugated DNA-based conformational switches that, upon binding to a target antibody, can trigger the cell-free transcription of a light-up fluorescence-activating RNA aptamer. The system couples the unique programmability and responsiveness of DNA-based systems with the specificity and sensitivity offered by in-vitro genetic circuitries and commercially available transcription kits. We demonstrate that cell-free transcriptional switches can efficiently measure antibody levels directly in blood serum. Thanks to the programmable nature of the sensing platform the method can be adapted to different antibodies: we demonstrate here the sensitive, rapid and cost-effective detection of three different antibodies and the possible use of this approach for the simultaneous detection of two antibodies in the same solution.
\end{abstract}




\section{Introduction}

Diagnostic tests that are easy to perform, convenient, reliable and suitable for use at the point-of-care are crucially needed in the detection, monitoring and containment of infectious diseases and other clinical emergencies. ${ }^{1-4}$ In recent years, the possibility to couple the advantages of synthetic nucleic acids (i.e., programmability of interactions, low-cost and ease of synthesis) together with the sensitivity offered by cell-free transcription-translation systems has lead to the development of innovative sensors for the detection of different targets. ${ }^{5-14}$ Synthetic genetic circuits and switches that respond to specific DNA or RNA sequences ${ }^{5-8}$, small molecules $^{9,10}$ and metal ions ${ }^{11}$ and trigger the cell-free transcription of signalling RNA aptamers or the translation of reporter proteins have led to analytical devices with excellent sensitivities and specificities. Recently, cell-free diagnostic tools for the detection of SARS-CoV-2 viral RNA fragments have been found useful even in the current COVID-19 pandemy. 13,14

Despite the above advances, the examples reported so far of cell-free nucleic-acid diagnostics have been demonstrated for a limited number of targets. The potentialities offered by responsive nucleic acid devices have thus not yet been fully exploited. Synthetic nucleic acid strands can be used as molecular scaffolds to append different recognition elements that allow the design of nucleic acid probes responsive to a wide range of targets. ${ }^{15-19}$ In a demonstration of such potentiality, we and others have recently employed antigen-conjugated nucleic acids rationally designed to respond to clinically-relevant antibodies. ${ }^{19-23}$

Motivated by the above considerations, we demonstrate here a cell-free diagnostic platform for the detection of specific antibodies in blood serum based on the use of antibody-responsive nucleic acid transcriptional switches. The approach we propose couples the advantageous features of responsive DNA-based conformational switching probes with those of cell-free diagnostic methods in which target-induced transcription/translation of signalling RNA aptamers or proteins is used for detection purposes. 


\section{Results and discussion}

\section{Sensing principle of antibody-responsive transcriptional switches.}

Our strategy to achieve an antibody-responsive transcriptional switch is based on the use of a pair of DNA-based functional modules that are rationally designed to trigger the transcription of a signalling RNA aptamer in the presence of a specific target antibody (Fig. 1a). The first module of this platform is the transcriptional switch, a conformational-switching hairpin DNA composed of two complementary DNA strands (Fig. 1a). Such transcriptional switch consists of three major domains: a double-stranded (ds) portion encoding for the RNA output of the switch (red domain), a T7 RNA Polymerase (RNAP) promoter domain (blue) and a switching domain encoded in the stem-loop structure of the hairpin. The output of the transcriptional switch is a light-up RNA aptamer such as Mango or Spinach aptamer. ${ }^{24-26}$ The switch is designed so that in the absence of the specific target antibody the transcription of the output RNA aptamer is inefficient due to the incomplete nature of the promoter domain.

The second module of the platform is the antibody-responsive module composed of two antigen-conjugated DNA strands (here also named split input strands). These strands are rationally designed so that hybridization of their complementary portions (black, Fig. 1a) leads to the formation of a bimolecular functional complex (input) that is able to induce a conformational change in the transcriptional switch through a toehold strand displacement reaction. Such conformational change leads to the formation of a complete promoter domain that can be recognized by T7-RNAP and induce efficient transcription (Fig. 1a, right). The complementary portions of the antigen-conjugated DNA strands, however, are designed to form an unstable complex under the diluted concentrations employed. Only upon bivalent binding of the target antibody to these two strands, a colocalization-induced formation of the functional complex (input) is achieved ultimately triggering the reaction with the transcriptional switch (Fig. 1b). The modules can be used with a commercially available cell-free transcription kit (containing T7-RNAP, the nucleotides and other components) and the fluorescent signal generated in the presence of the aptamer-binding dye will inform on the presence and concentration of the target antibody (Fig. 1b). 

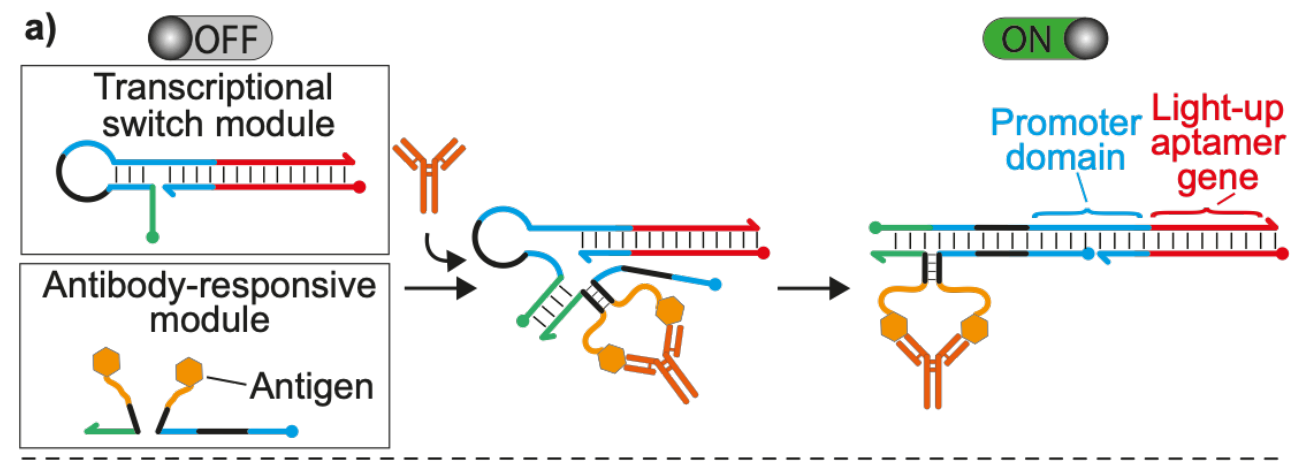

b)

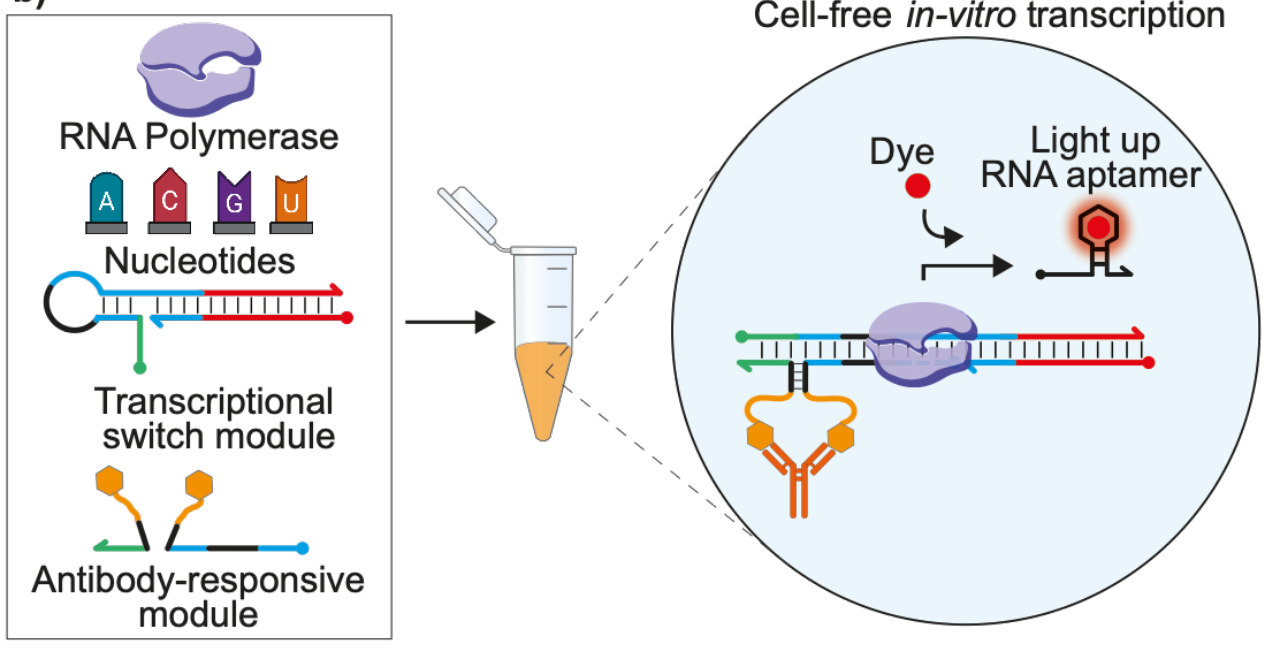

Figure 1. Programmable antibody-responsive transcriptional switches. a) The antibodyresponsive transcriptional switch is composed of two modules: the transcriptional switch module and the antibody-responsive module. The first is a double-stranded DNA switch designed to adopt a stem-loop hairpin conformation that prevents efficient transcription of an RNA light-up aptamer due to incomplete formation of the promoter sequence. The second module is composed of two antigen-conjugated DNA strands (split input strands) that, upon bivalent binding to a target antibody, are brought into close proximity and can hybridize to form a functional bimolecular complex. Such complex induces a conformational change on the switch and makes the promoter sequence accessible for transcription (right). b) The so-activated transcriptional switch can transcribe, in the presence of RNA polymerase and nucleotides, a reporter light-up RNA aptamer that signals the presence of the target antibody.

\section{Design of transcriptional switches.}

We initially focused on the thermodynamic optimization of the transcriptional switch module. Instrumental for the functioning of the sensing system is the need to have a switch that only induces transcription of the light-up RNA aptamer upon a strand displacement reaction with a functional input strand. We designed a set of transcriptional switches that share the same 17-nt T7 RNAP promoter sequence and the same hairpin structure with a 11-nt loop and a 6-nt stem (Fig. 2a,S1). The variants we designed have a variable length of the promoter sequence hidden in the stem-loop structure (from 1 to $17 \mathrm{nt}$ ) (Fig. $2 \mathrm{~b}$ and in Fig. 2c three variants are shown as 
an example). As the reporter light-up RNA aptamer produced by the transcriptional switch we first employed the well-characterized Mango aptamer, a 39-nt RNA sequence that binds to a thiazole orange (TO-1) derivative resulting in an increase of its fluorescence signal. ${ }^{24-26} \mathrm{We}$ tested the Mango aptamer signal in the absence and presence of a linear unimolecular DNA input strand that mimics the input strand that will be formed upon bivalent antibody binding to the antibody-responsive module (Fig. 2a). We observed no signalling in the absence of the input strand with transcriptional switches in which the length of the accessible promoter domain is shorter than $12 \mathrm{nt}$. By further increasing the length of the accessible promoter domain we observe higher signals that level off when this reaches $14 \mathrm{nt}$. In the presence of the input strand, instead, efficient transcription is observed for all switches with accessible promoter domains longer than $8 \mathrm{nt}$. For shorter accessible promoter domains no significant transcription is observed even after the input-induced conformational switch, likely because the presence of the nick in the formed promoter affects efficient T7-RNAP transcription. ${ }^{27}$ We found that a transcriptional switch with a $10 \mathrm{nt}$ accessible promoter domain leads to the highest increase in Mango aptamer transcription in the presence of the input strand (Fig. 2c).

With this optimized transcriptional switch we then moved to the demonstration of the colocalization-induced transcription. To do this we performed transcription experiments in the presence of the two strands composing the antibody-responsive module (i.e., split input strands). A 6-nt complementary portion between the two DNA strands was used (Fig. 2g) as this length was previously demonstrated optimal to observe colocalization-induced hybridization. ${ }^{28,29}$ For this initial proof of principle, we used unmodified split input strands. As expected, under diluted conditions ( $<30 \mathrm{nM}$ ) the two strands do not lead to efficient transcription of the Mango aptamer and only at saturating concentrations $(\geq 100 \mathrm{nM})$ we observe a signal comparable to that observed with an unimolecular input strand (Fig. 2h). We then employed a bivalent DNA strand that acts as an antibody-mimic (Ab-mimic) and binds the tails of the two split input strands in a way similar to that expected from the binding of a bivalent antibody (Fig. 2g). This Ab-mimic strand produces light-up RNA aptamer with an efficiency similar to that of the complete unimolecular input strand even under diluted conditions (30 $\mathrm{nM})$ thus supporting the proposed sensing scheme (Fig. 2h-i,S2). 
a)

Accessible promoter

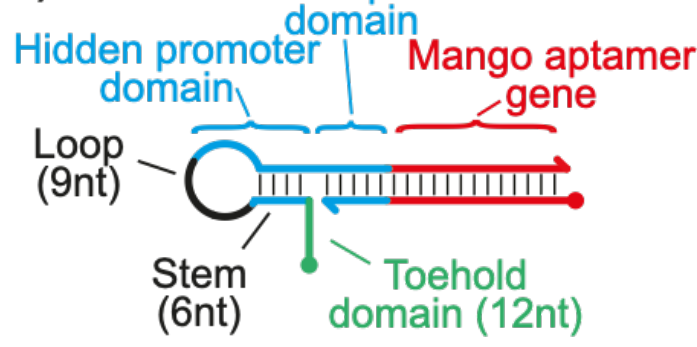

b)

\begin{tabular}{|c|c|c|c|c|c|c|c|c|c|}
\hline Variant \# & 1 & 2 & 3 & 4 & 5 & 6 & 7 & 8 & 9 \\
\hline $\begin{array}{l}\text { Accessible } \\
\text { promoter } \\
\text { domain (nt) }\end{array}$ & 0 & 2 & 4 & 6 & 8 & 10 & 12 & 14 & 416 \\
\hline $\begin{array}{l}\text { Hidden } \\
\text { promoter } \\
\text { domain (nt) }\end{array}$ & 17 & 15 & 13 & 11 & 9 & 7 & 5 & 3 & 1 \\
\hline
\end{tabular}

c)

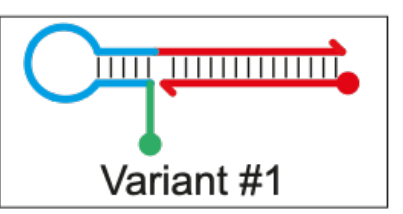

d)

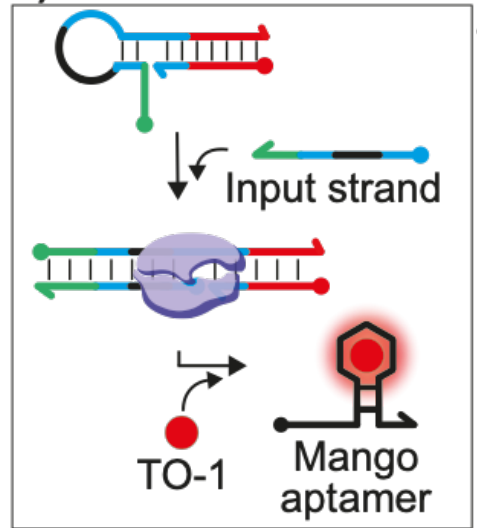

g)

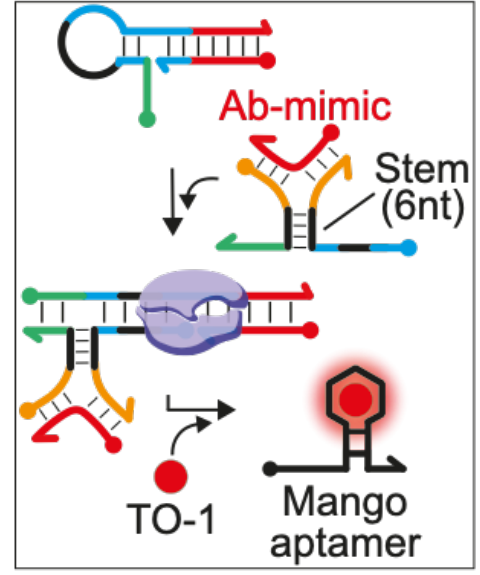

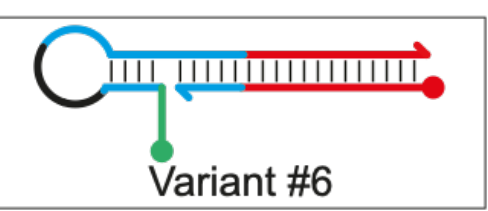

e)

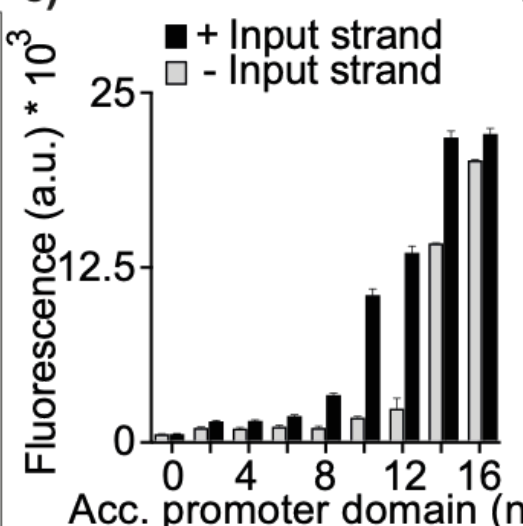

h)

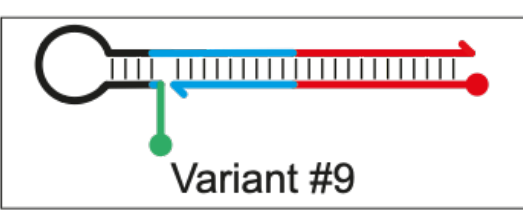

f)

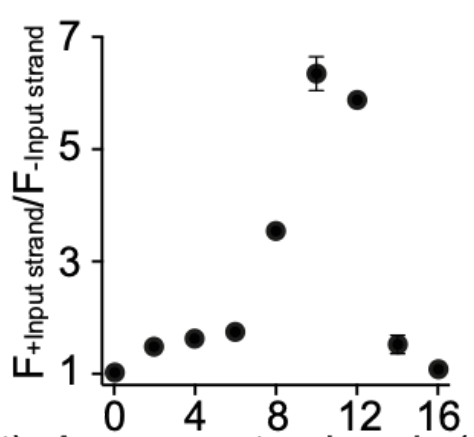

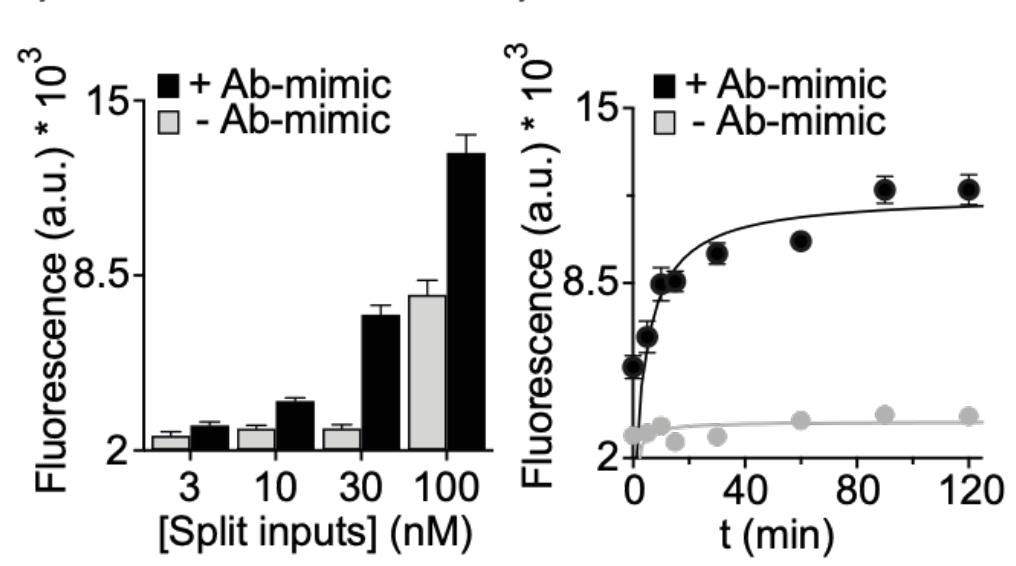

Figure 2. Design of colocalization-induced transcriptional switch. a) Scheme of the transcriptional switch with relevant functional domains indicated. b) Table of the different variants tested and their corresponding length of the accessible and hidden promoter domain. c) Three representative transcriptional switch variants (\#1, \#6 and \#9). d) Scheme of the strand displacement reaction between the transcriptional switch and an unimolecular input strand. e) Fluorescence signal obtained with the different variants in the absence and in the presence (30 $n M)$ of the unimolecular input strand. f) Ratio between the end-point fluorescence signals in the presence and absence of the input strand with the different variants. g) Scheme of the colocalization induced hybridization of the split input strands in the presence of an Ab-mimic DNA strand. h) Fluorescence signal in the absence and presence (100 nM) of the Ab-mimic DNA 
strand at different split input strands concentrations. i) Transcription kinetic traces in the absence (grey) and presence (black) of the Ab-mimic strand with a $30 \mathrm{nM}$ concentration of split input strands. The experiments here were conducted at $25^{\circ} \mathrm{C}$ in a $20 \mu \mathrm{L}$ solution of a commercial transcription kit supplemented with the transcriptional switch module (100 nM) and the split input strands (30 $\mathrm{nM}$ ) and the input strand (or Ab-mimic) were indicated. The transcription reaction was allowed to proceed for $120 \mathrm{~min}$ and then an aliquot was transferred to $100 \mu \mathrm{L}$ of $10 \mathrm{mM}$ Tris- $\mathrm{HCl}$ and $75 \mathrm{mM} \mathrm{KCl}, \mathrm{pH} 7.4$ solution containing $300 \mathrm{nM}$ of TO-1 and the fluorescence signal measured after $15 \mathrm{~min}$ at $545 \mathrm{~nm}$.

\section{Programmable transcriptional switches for antibodies detection.}

We then sought to demonstrate a proof of principle of transcriptional switches for antibodies detection. To do this we first employed as recognition element (i.e., antigen) the small hapten digoxigenin (Dig) and we conjugated it at the two ends of the antibody-responsive module strands (Fig. 3a). We performed transcription reactions with the transcriptional Mango switch module (100 nM) and the two Dig-conjugated DNA strands (at $30 \mathrm{nM}$ ) in the absence and presence of Anti-Dig monoclonal antibodies (Fig. 3a). First, we observe that efficient Mango aptamer transcription is achieved only in the presence of Anti-Dig antibodies (100 nM) with output signals that start to level off after 120 minutes of reaction (Fig. 3b). The fluorescence spectra obtained at the end of the transcription reaction in the presence of the Anti-Dig antibody show, as expected, the increase of the emission signal characteristic of the TO-1 dye when bound to the Mango aptamer (Fig. 3c). The platform responds in a concentration-dependent fashion to Anti-Dig antibodies generating a 4-fold increase in fluorescence at saturating concentration of Anti-Dig antibodies (100 nM, Fig. S3) and achieving a detection limit in the low nanomolar range $\left(K_{D}=9.7 \pm 0.4 \mathrm{nM}\right.$; Fig. S3). Of note, the transcriptional switch shows excellent sensitivities also in $10 \%$ bovine serum supplemented with increasing concentrations of Anti-Dig antibodies (Fig. 3d). The platform is also highly specific and no significant signal is observed in the presence of non-specific antibodies (at $100 \mathrm{nM}$ ) or in the presence of Anti-Dig Fab fragment (that, being monovalent, cannot induce co-localization of the two antigen-conjugated strands) (Fig. 3e).

One of the major advantages of our sensing strategy is that it is modular and versatile: changing the recognition elements allows us to potentially detect different target antibodies. To demonstrate this we have employed responsive DNA strands conjugated with another recognition element (i.e., dinitrophenol, DNP) and designed a second transcriptional switch programmed to transcribe the Spinach aptamer as the reporter aptamer. With this new antibodyresponsive transcriptional switch we have measured Anti-DNP antibodies (lgG) (Fig. 3f) 
reaching sensitivities and specificities similar to those observed for the Anti-Dig antibodies (Fig. $3 g-1, S 4)$.

Because the two antibody-responsive transcriptional switches we have characterized specifically respond to a different antibody and induce the transcription of a specific light-up aptamer, we can use them in the same solution to achieve simultaneous detection of two antibodies. To do this we mixed in the same solution the two transcriptional switches and added either one of the two antibodies or both of them (Fig. S5). While the presence of a single antibody induces the fluorescence signal increase of the relevant dye-binding RNA aptamer, we observe high fluorescence signals for both dyes only in the presence of both antibodies (Fig. S5).
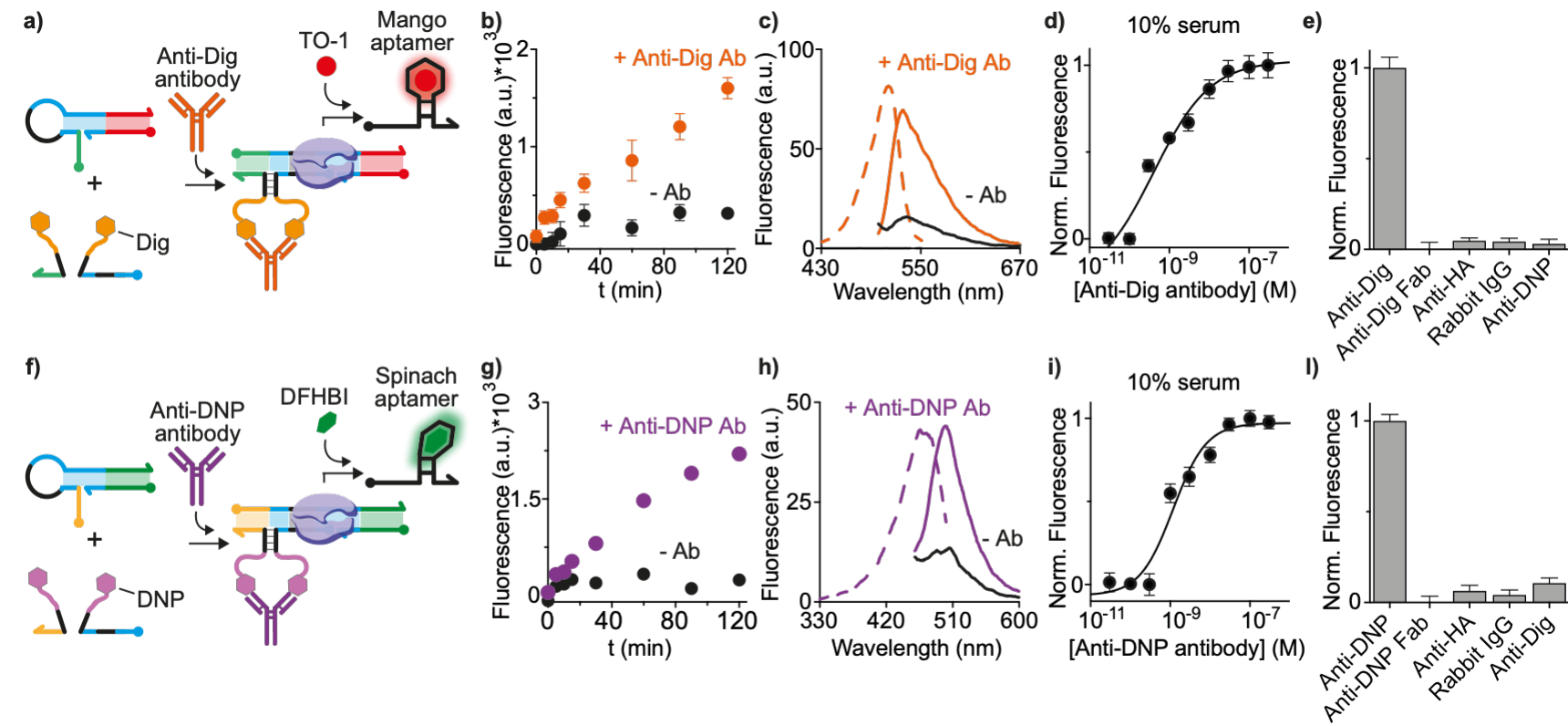

Figure 3. Antibody detection using programmable antibody-responsive transcriptional switches. a) Scheme of the transcriptional switch for the detection of Anti-Dig antibodies. b) Time course experiments showing the signal of the Mango aptamer-binding fluorophore (TO-1) obtained upon cell-free transcription experiments carried out in the absence and presence (100 $n M)$ of Anti-Dig antibody. c) Emission and excitation spectra at the end-point (120 min) of reactions shown in panel b. d) Fluorescence signals in a $10 \%$ diluted bovine serum solution supplemented with increasing Anti-Dig antibody concentrations. e) Fluorescence signal obtained with Anti-Dig antibodies and different non-specific targets (all at $100 \mathrm{nM}$ ). f) Scheme of the transcriptional switch activated by Anti-DNP antibody using DNP-conjugated DNA strands. g) Time course experiments showing the signal of the Spinach aptamer-binding fluorophore (DFHBI) obtained upon cell-free transcription experiments carried out in the absence and presence (100 nM) of Anti-DNP antibody. h) Emission and excitation spectra at the end-point (120 min) of the reactions shown in panel g. i) Fluorescence signals in a $10 \%$ diluted bovine serum solution supplemented with increasing Anti-DNP antibody concentrations. I) Fluorescence signal obtained with Anti-DNP antibodies and different non-specific targets (all at 
$100 \mathrm{nM})$. The experiments here were conducted at $25^{\circ} \mathrm{C}$ in a $20 \mu \mathrm{L}$ solution of a commercial transcription kit supplemented with the transcriptional switch module (100 nM), the antibodyresponsive module (30 $\mathrm{nM}$ ) and the antibody (at the indicated concentration). The transcription reaction was allowed to proceed for $120 \mathrm{~min}$ and then an aliquot was transferred to $100 \mu \mathrm{L}$ of $10 \mathrm{mM}$ Tris- $\mathrm{HCl}$ and $75 \mathrm{mM} \mathrm{KCl}, \mathrm{pH} 7.4$ solution containing the relevant dye and the fluorescence signal measured after $15 \mathrm{~min}$.

\section{Modular transcriptional switch for antibodies detection.}

After proof of principle demonstration of our transcriptional antibody-responsive switch we sought to expand it to more clinically-relevant antibodies. In this regard it should be considered that antibodies produced upon infection are commonly raised against proteins epitopes or peptides. ${ }^{30,31}$ From a synthetic point of view, the conjugation of peptides (and whole proteins) to DNA strands can be challenging and expensive. To overcome this limitation, we designed a modular version of our antibody-responsive transcriptional switch that can be easily adapted to the use of peptides as recognition elements. To do this we re-engineered the two antibodyresponsive module strands to contain a 17 -nt tail domain that can hybridize to a peptide-PNA strand (this presents a lower degree of difficulty of synthesis) (Fig. 4a). With this modular approach we have employed as recognition element a 9-residue epitope enclosed in the human influenza hemagglutinin (HA) protein ${ }^{32}$ and recognized by Anti-HA antibody (Fig. 4a). This modular switch achieves sensitivities and specificities similar to its non-modular counterpart. More specifically we found a sensitivity in the nanomolar range $\left(K_{D}=5 \pm 1 \mathrm{nM}\right.$ in buffer, $K_{D}=$ $4.1 \pm 0.2 \mathrm{nM}$ in 10\% serum) (Fig. 4b, S6-7) and no signal change in the presence of non-specific antibodies (Anti-Dig, Anti-DNP and Rabbit lgG) (Fig. S8). We have also evaluated the accuracy of the proposed method by determining the recovery percentages of spiked serum samples at four representative Anti-HA concentration levels $(2,7,17$ and $25 \mathrm{nM}, \mathrm{n}=10$ for each concentration) (Fig. 4c). The results indicated good accuracy with recovery percentages between $77 \%$ (at $2 \mathrm{nM}$ Anti-HA concentration, C.I. $95 \%=1.4$ to $1.7 \mathrm{nM}$ ) and $107 \%$ (at $7 \mathrm{nM}$ AntiHA concentration, C.I. 95\% $=6.3-8.1 \mathrm{nM})$ (Fig. 4c, S9). 


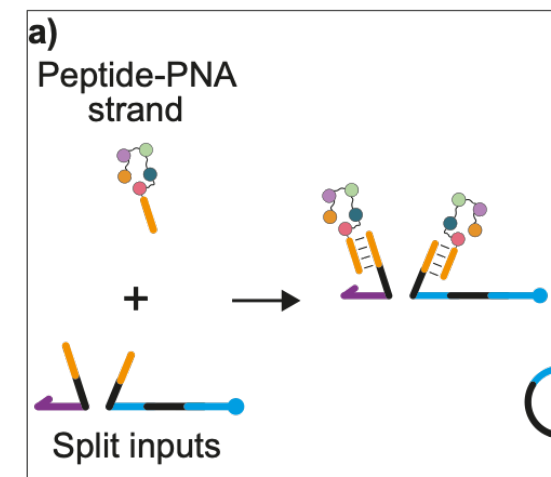

b)

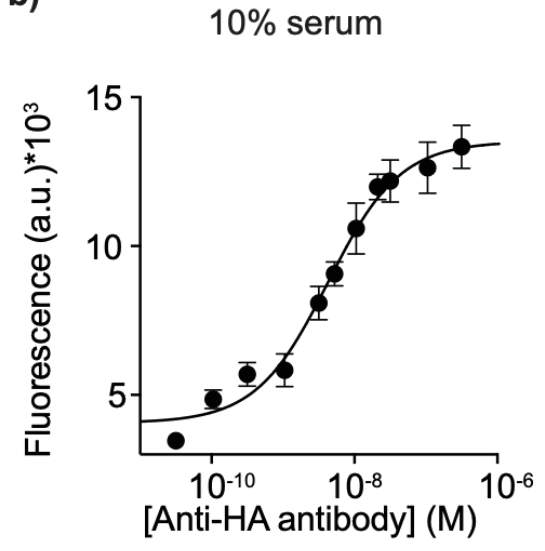

Light up

Target antibody

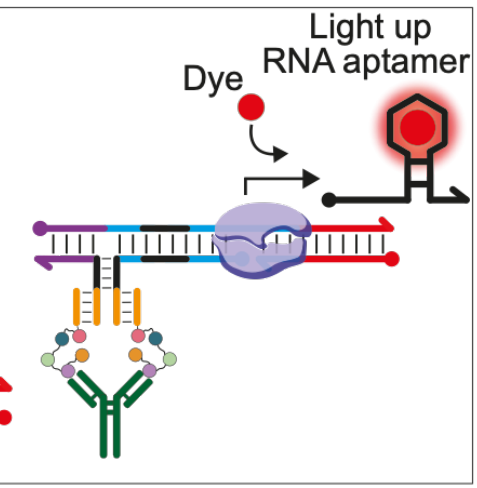

c)

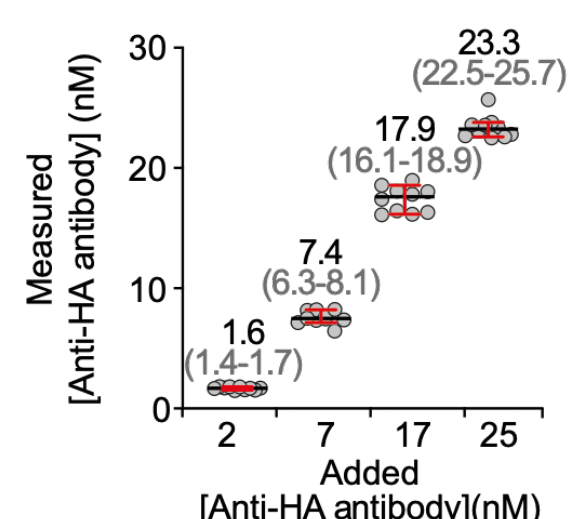

[Anti-HA antibody](nM)

Figure 4. Modular transcriptional switch for Anti-HA antibodies detection. a) Modular version of the transcriptional switch to use peptide-PNA chimera strands as recognition elements of Anti-HA antibodies. b) Fluorescence signal as a function of Anti-HA concentration in a $10 \%$ diluted bovine serum solution. c) Antibody quantification assessed by spiking blank sera samples with different known concentrations of Anti-HA antibody (2, 7, 17, and $25 \mathrm{nM}$ ). Median values (black value and horizontal line) and 95\% confidence intervals (grey values in parenthesis and red bars) are indicated. The experiments here were conducted at $25^{\circ} \mathrm{C}$ in a 20 $\mu L$ solution of a commercial transcription kit supplemented with the transcriptional switch module (100 nM), split input strands (30 nM), HA-PNA chimera strands (100 nM) and the Anti-HA antibody. The transcription reaction was allowed to proceed for 120 min and then an aliquot was transferred to $100 \mu \mathrm{L}$ of $10 \mathrm{mM}$ Tris- $\mathrm{HCl}$ and $75 \mathrm{mM} \mathrm{KCl}, \mathrm{pH} 7.4$ solution containing $300 \mathrm{nM}$ of TO-1 and the fluorescence signal measured after $15 \mathrm{~min}$. 


\section{Conclusion}

In the present study we report the development of cell-free transcriptional switches that can be activated by specific target antibodies. Our platform consists of DNA programmable modules that can respond to the presence of a target antibody and trigger the transcription of a light-up RNA aptamer. The modular nature of our platform makes it easily applicable to different antibodies with the expedient of changing the recognition element conjugated to the DNA responsive module. By using this approach, we demonstrated efficient detection of three different antibodies with high sensitivity (low nanomolar levels), excellent specificity (no significant cross-reactivity with non-specific antibodies) and rapid response time. The transcriptional switch can also efficiently detect antibodies in complex biological matrices, such as blood serum. Thanks to the programmability of DNA-DNA interactions it is also possible to design orthogonal modules that can respond to different target antibodies in the same solution.

This work builds on previous advancements in the field of cell-free nucleic-acid diagnostics ${ }^{5-}$ 14 and demonstrates that transcription/translation in-vitro processes combined with programmable responsive nucleic acid devices can be conveniently applied for the detection of a wide range of clinical markers. Considering the importance of the detection of antibodies in controlling infectious diseases and their growing therapeutic use, ${ }^{33-35}$ this and similar approaches can lead to transformative improvements in antibody-detection technologies applied to clinical problems. 


\section{Experimental section}

Reagents and Materials. Reagent-grade chemicals $\left(\mathrm{NaCl}, \mathrm{KCl}, \mathrm{MgCl}_{2}\right.$, Trizma base, Trizma hydrochloride, DEPC treated water) were purchased from Sigma-Aldrich (St Louis, Missouri) and used without further purifications. TO-1-3PEG-Biotin (TO-1) fluorophore was purchased from abmgood (Canada, Cat \#G955), DFBHI-1T was purchased from TOCRIS biosciences (Bristol, UK) Cat. No. 1625/10. Sheep polyclonal Anti-Dig antibodies were purchased from Roche Diagnostic Corporation, Germany, (cat\#: 11333089001), mouse monoclonal Anti-DNP antibodies and rabbit polyclonal Anti-mouse lgG antibodies were purchased from Sigma-Aldrich, USA, (cat\#: D9656, cat\# 06-371), Anti-Dig Fab and Anti-HA antibodies were purchased from Roche Diagnostic Corporation, (Germany) (cat\#: 11214667001). All the antibodies were aliquoted and stored at $4{ }^{\circ} \mathrm{C}$ for immediate use or at -20 ${ }^{\circ} \mathrm{C}$ for long-term storage.

Oligonucleotides. HPLC purified oligonucleotides were purchased from Metabion, (Planegg, Germany) or Biosearch Technologies (Risskov, Denmark). The split input oligonucleotide strands that activate the transcriptional switches were modified with digoxigenin or dinitrophenyl moieties. PNA sequences modified with HA peptide were obtained from Panagene (South Korea). All the sequences of the transcriptional switch variants and antibodyresponsive modules are reported in the Supplementary Information document.

Cell free transcription reactions. All transcription reactions were performed using the ThermoFisher TranscriptAid T7 high yield Transcription kit (ref. K0441) following the recommended manufacturer protocols. More specifically, we have prepared the $20 \mu \mathrm{L}$ solution of the commercial transcription kit so that they contain the transcriptional switch module (100 $\mathrm{nM}$ ), the antibody-responsive module (30 nM) and the antibody (when indicated). The transcription reaction was allowed to proceed for $120 \mathrm{~min}$ after polymerase addition. An aliquot of the transcription reaction solution $(1 \mu \mathrm{L}$ or $5 \mu \mathrm{L}$ ) was then transferred to $100 \mu \mathrm{L}$ of $10 \mathrm{mM}$ Tris$\mathrm{HCl}$ and $75 \mathrm{mM} \mathrm{KCl}, \mathrm{pH} 7.4$ solution containing the relevant dye (either TO-1, $300 \mathrm{nM}$ or DFHBI, $\mu 1 \mathrm{M})$ and the fluorescence signal measured after $15 \mathrm{~min}$.

Fluorescence experiments. Fluorescence measurements were obtained in a Tecan M200pro plate reader using top reading mode with black, flat bottom non-binding 96 well plates. For Mango RNA aptamer (TO-1 dye) excitation wavelength was fixed at $506( \pm 9) \mathrm{nm}$ and 
emission wavelength at $545( \pm 20) \mathrm{nm}$. For the Spinach RNA aptamer (DFHBI dye) excitation wavelength was fixed at $455( \pm 9) \mathrm{nm}$ and emission wavelength at $506( \pm 20) \mathrm{nm}$. Curve fitting at different concentrations of target antibody were obtained using Prism-GraphPad software and its built-in Hill function with the following Lavenberg Marquardt iteration algorithm:

$$
\mathrm{F}=F_{0}+\Delta \mathrm{F} \frac{[\text { Target }]}{\mathrm{K}_{\mathrm{D}}+[\text { Target }]}
$$

Where $F_{0}$ is the fluorescence background, $\Delta F$ is the fluorescence signal change and $K_{D}$ is the equilibrium concentration at half-maximum signal. To allow for more ready interpretation of the results, fluorescence signals obtained have been normalized on a $0-1$ scale using the following formula:

$$
\text { Norm. Fluo. }=\frac{\left[\mathrm{F}_{T}-F_{0}\right]}{\left[\mathrm{F}_{\text {Max }}-F_{0}\right]}
$$

where $F_{T}$ is the fluorescence signal obtained in presence of the target antibody, $F_{0}$ is the fluorescence signal obtained in the absence of target and $F_{\max }$ represents the maximum fluorescence signal of the platform at a saturating concentration of target (Rel. Fluor. $=1$ ). The experimental values represent averages of three separate measurements and the error bars reflect the standard deviations.

Anti-HA antibody quantification. Recovery experiments were performed adding known amounts of Anti-HA antibody into blank serum samples. Anti-HA antibody quantification was performed through a linear regression analysis by tracing the intercept on the $x$-axis a value corresponding to the concentration of the antibody. Differences between spiked serum concentrations were calculated with a two-ways ANOVA test.

\section{Acknowledgment}

This work was in part supported by Associazione Italiana per la Ricerca sul Cancro, AIRC (project n. 14420) (F.R.), by the European Research Council, ERC (Consolidator Grant project n. 819160) (F.R.), by the Marie Skłodowska-Curie grant agreement ("DNA-bots" project $n$. 843998) (T.P.), by the Marie Skłodowska-Curie grant agreement ("DNA-NANO-AB" project $n$. 
843179) (S.R.) and by the Marie Skłodowska-Curie ITN project DNA-robotics ("DNA-robotics", project n. 765703) (F.R. and A.P.D.).

\section{References}

(1) Wiersinga, W. J.; Rhodes, A.; Cheng, A. C.; Peacock, S. J.; Prescott, H. C. Pathophysiology, Transmission, Diagnosis, and Treatment of Coronavirus Disease 2019 (COVID-19): A Review. JAMA 2020, 324 (8), 782.

(2) Moody, A. Rapid Diagnostic Tests for Malaria Parasites. Clin. Microbiol. Rev. 2002, 15 (1), $66-78$.

(3) Li, Z.; Yi, Y.; Luo, X.; Xiong, N.; Liu, Y.; Li, S.; Sun, R.; Wang, Y.; Hu, B.; Chen, W.; Zhang, Y.; Wang, J.; Huang, B.; Lin, Y.; Yang, J.; Cai, W.; Wang, X.; Cheng, J.; Chen, Z.; Sun, K.; Pan, W.; Zhan, Z.; Chen, L.; Ye, F. Development and Clinical Application of a Rapid IgMIgG Combined Antibody Test for SARS-CoV-2 Infection Diagnosis. J. Med. Virol. 2020, 92 (9), 1518-1524.

(4) Loizou, S.; McCrea, J. D.; Rudge, A. C.; Reynolds, R.; Boyle, C. C.; Harris, E. N. Measurement of Anti-Cardiolipin Antibodies by an Enzyme-Linked Immunosorbent Assay (ELISA): Standardization and Quantitation of Results. Clin. Exp. Immunol. 1985, 62 (3), 738-745.

(5) Pardee, K.; Green, A. A.; Takahashi, M. K.; Braff, D.; Lambert, G.; Lee, J. W.; Ferrante, T.; Ma, D.; Donghia, N.; Fan, M.; Daringer, N. M.; Bosch, I.; Dudley, D. M.; O’Connor, D. H.; Gehrke, L.; Collins, J. J. Rapid, Low-Cost Detection of Zika Virus Using Programmable Biomolecular Components. Cell 2016, 165 (5), 1255-1266.

(6) Gootenberg, J. S.; Abudayyeh, O. O.; Kellner, M. J.; Joung, J.; Collins, J. J.; Zhang, F. Multiplexed and Portable Nucleic Acid Detection Platform with Cas13, Cas12a, and Csm6. Science 2018, 360 (6387), 439-444.

(7) Pardee, K.; Green, A. A.; Ferrante, T.; Cameron, D. E.; DaleyKeyser, A.; Yin, P.; Collins, J. J. Paper-Based Synthetic Gene Networks. Cell 2014, 159 (4), 940-954.

(8) Slomovic, S.; Pardee, K.; Collins, J. J. Synthetic Biology Devices for in Vitro and in Vivo Diagnostics. Proc. Natl. Acad. Sci. USA 2015, 112 (47), 14429.

(9) Sadat Mousavi, P.; Smith, S. J.; Chen, J. B.; Karlikow, M.; Tinafar, A.; Robinson, C.; Liu, W.; Ma, D.; Green, A. A.; Kelley, S. O.; Pardee, K. A Multiplexed, Electrochemical Interface for Gene-Circuit-Based Sensors. Nat. Chem. 2020, 12 (1), 48-55. 
(10) Silverman, A. D.; Akova, U.; Alam, K. K.; Jewett, M. C.; Lucks, J. B. Design and Optimization of a Cell-Free Atrazine Biosensor. ACS Synth. Biol. 2020, 9 (3), 671-677.

(11) Jung, J. K.; Alam, K. K.; Verosloff, M. S.; Capdevila, D. A.; Desmau, M.; Clauer, P. R.; Lee, J. W.; Nguyen, P. Q.; Pastén, P. A.; Matiasek, S. J.; Gaillard, J.-F.; Giedroc, D. P.; Collins, J. J.; Lucks, J. B. Cell-Free Biosensors for Rapid Detection of Water Contaminants. Nat. Biotechnol. 2020, 38 (12), 1451-1459.

(12) Tan, X.; Letendre, J. H.; Collins, J. J.; Wong, W. W. Synthetic Biology in the Clinic: Engineering Vaccines, Diagnostics, and Therapeutics. Cell 2021, 184 (4), 881-898.

(13) Nguyen, P. Q.; Soenksen, L. R.; Donghia, N. M.; Angenent-Mari, N. M.; de Puig, H.; Huang, A.; Lee, R.; Slomovic, S.; Galbersanini, T.; Lansberry, G.; Sallum, H. M.; Zhao, E. M.; Niemi, J. B.; Collins, J. J. Wearable Materials with Embedded Synthetic Biology Sensors for Biomolecule Detection. Nat. Biotechnol. 2021, 1-9.

(14) de Puig, H.; Lee, R. A.; Najjar, D.; Tan, X.; Soeknsen, L. R.; Angenent-Mari, N. M.; Donghia, N. M.; Weckman, N. E.; Ory, A.; Ng, C. F.; Nguyen, P. Q.; Mao, A. S.; Ferrante, T. C.; Lansberry, G.; Sallum, H.; Niemi, J.; Collins, J. J. Minimally Instrumented SHERLOCK (MiSHERLOCK) for CRISPR-Based Point-of-Care Diagnosis of SARS-CoV2 and Emerging Variants. Sci. Adv. 2021, 7 (32), eabh2944.

(15) Ranallo, S.; Porchetta, A.; Ricci, F. DNA-Based Scaffolds for Sensing Applications. Anal. Chem. 2019, 91 (1), 44-59.

(16) Claudio Parolo; Greenwood, A. S.; Ogden, N. E.; Kang, D.; Hawes, C.; Ortega, G.; ArroyoCurrás, N.; Plaxco, K. W. E-DNA Scaffold Sensors and the Reagentless, Single-Step, Measurement of HIV-Diagnostic Antibodies in Human Serum. Microsyst. Nanoeng. 2020, $6(1), 1-8$.

(17) Chen, X.; Zhou, G.; Song, P.; Wang, J.; Gao, J.; Lu, J.; Fan, C.; Zuo, X. Ultrasensitive Electrochemical Detection of Prostate-Specific Antigen by Using Antibodies Anchored on a DNA Nanostructural Scaffold. Anal. Chem. 2014, 86 (15), 7337-7342.

(18) Janssen, B. M. G.; van Rosmalen, M.; van Beek, L.; Merkx, M. Antibody Activation Using DNA-Based Logic Gates. Angew. Chem. Int. Ed. Engl. 2015, 127 (8), 2560-2563.

(19) a) Porchetta, A.; Ippodrino, R.; Marini, B.; Caruso, A.; Caccuri, F.; Ricci, F. Programmable Nucleic Acid Nanoswitches for the Rapid, Single-Step Detection of Antibodies in Bodily Fluids. J. Am. Chem. Soc. 2018, 140 (3), 947-953. b) Bracaglia, S.; Ranallo, S.; Plaxco, K. W.; Ricci, F. Programmable, Multiplexed DNA Circuits Supporting Clinically Relevant, Electrochemical Antibody Detection. ACS Sens. 2021, 6 (6), 2442-2448. 
(20) Engelen, W.; Meijer, L. H. H.; Somers, B.; de Greef, T. F. A.; Merkx, M. Antibody-Controlled Actuation of DNA-Based Molecular Circuits. Nat. Commun. 2017, 8, 14473.

(21) Rossetti, M.; Brannetti, S.; Mocenigo, M.; Marini, B.; Ippodrino, R.; Porchetta, A. Harnessing Effective Molarity to Design an Electrochemical DNA-Based Platform for Clinically Relevant Antibody Detection. Angew. Chem. Int. Ed. Engl. 2020, 59 (35), 1497314978.

(22) Tomimuro, K.; Tenda, K.; Ni, Y.; Hiruta, Y.; Merkx, M.; Citterio, D. Thread-Based Bioluminescent Sensor for Detecting Multiple Antibodies in a Single Drop of Whole Blood. ACS Sens. 2020, 5 (6), 1786-1794.

(23) Tenda, K.; van Gerven, B.; Arts, R.; Hiruta, Y.; Merkx, M.; Citterio, D. Paper-Based Antibody Detection Devices Using Bioluminescent BRET-Switching Sensor Proteins. Angew. Chem. Int. Ed. Engl. 2018, 57 (47), 15369-15373.

(24) Dolgosheina, E. V.; Jeng, S. C. Y.; Panchapakesan, S. S. S.; Cojocaru, R.; Chen, P. S. K.; Wilson, P. D.; Hawkins, N.; Wiggins, P. A.; Unrau, P. J. RNA Mango Aptamer-Fluorophore: A Bright, High-Affinity Complex for RNA Labeling and Tracking. ACS Chem. Biol. 2014, 9 (10), 2412-2420. h

(25) Ouellet, J. RNA Fluorescence with Light-Up Aptamers. Front. Chem. 2016, 4, 29.

(26) Paige, J. S.; Wu, K. Y.; Jaffrey, S. R. RNA Mimics of Green Fluorescent Protein. Science 2011, 333 (6042), 642-646.

(27) Gong, P.; Martin, C. T. Mechanism of Instability in Abortive Cycling by T7 RNA Polymerase. J. Bio. Chem. 2006, 281 (33), 23533-23544.

(28) Ranallo, S.; Sorrentino, D.; Ricci, F. Orthogonal Regulation of DNA Nanostructure SelfAssembly and Disassembly Using Antibodies. Nat. Commun. 2019, 10 (1), 5509.

(29) Baranda Pellejero, L.; Mahdifar, M.; Ercolani, G.; Watson, J.; Brown, T.; Ricci, F. Using Antibodies to Control DNA-Templated Chemical Reactions. Nat. Commun. 2020, 11 (1), 6242.

(30) Ekiert, D. C.; Bhabha, G.; Elsliger, M.-A.; Friesen, R. H. E.; Jongeneelen, M.; Throsby, M.; Goudsmit, J.; Wilson, I. A. Antibody Recognition of a Highly Conserved Influenza Virus Epitope. Science 2009, 324 (5924), 246-251. h

(31) Murin, C. D.; Wilson, I. A.; Ward, A. B. Antibody Responses to Viral Infections: A Structural Perspective across Three Different Enveloped Viruses. Nat. Microbiol. 2019, 4 (5), 734 747.

(32) Fleury, D.; Wharton, S. A.; Skehel, J. J.; Knossow, M.; Bizebard, T. Antigen Distortion Allows Influenza Virus to Escape Neutralization. Nat. Struct. Biol. 1998, 5 (2), 119-123. 
(33) Ludwig, J. A.; Weinstein, J. N. Biomarkers in Cancer Staging, Prognosis and Treatment Selection. Nat. Rev. Cancer. 2005, 5 (11), 845-856.

(34) Cutts, J. C.; Powell, R.; Agius, P. A.; Beeson, J. G.; Simpson, J. A.; Fowkes, F. J. I. Immunological Markers of Plasmodium Vivaxexposure and Immunity: A Systematic Review and Meta-Analysis. BMC Med. 2014, 12 (1), 150.

(35) Gubala, V.; Harris, L. F.; Ricco, A. J.; Tan, M. X.; Williams, D. E. Point of Care Diagnostics: Status and Future. Anal. Chem. 2012, 84 (2), 487-515. 